\title{
Implante intra-miocárdico de artéria torácica interna: cirurgia de Vineberg
}

\author{
Gladyston Luiz Lima SOUTO*, Celme da S. CAETANO*, José B. de SOUZA*, Ary G. de PAULA*, \\ Hanry B. SOUTO*, Sandro B. P. COELHO*, Elisângela S. V. dos REIS*, Marco A. TEIXEIRA*, \\ Márcio R. M. de CARVALHO*, Antônio C. BOTELHO*
}

RBCCV 44205-514

\begin{abstract}
Souto G L L, Caetano C S, Souza J B, Paula A G, Souto H B, Coelho S B P, Reis E S V, Teixeira M A, Carvalho M R M, Botelho A C - Implante intra-miocárdico de artéria torácica interna: cirurgia de Vineberg. Rev Bras Cir Cardiovasc 2000; 15(4): 285-92.
\end{abstract}

RESUMO: Objetivos: Demonstrar nossa experiência com o implante da artéria torácica interna, para revascularização do miocárdio, procedimento proposto por Vineberg, em 1946.

Casuística e Método: De agosto de 1994 a setembro de 1998, foram operados 10 pacientes nos quais foi implantada a artéria torácica interna esquerda na parede ântero-lateral do ventrículo esquerdo. Em todos esses pacientes também foi feito enxerto de safena aorto-coronária. A idade dos pacientes variou de 42 a 73 anos, tendo sexo masculino prevalecido (60\%). Todos os pacientes apresentavam doença coronária arterosclerótica difusa, mas com miocárdio viável. A indicação para o implante de artéria torácica interna foi feita durante a operação, devido a impossibilidade de fazer enxerto ou endarterectomia.

Resultados: Todos os pacientes estavam muito bem no pós-operatório imediato. O reestudo angiográfico foi feito em 9 pacientes entre 45 dias e 23 meses de pós-operatório, mostrando patência da artéria implantada e uma vasta rede que se comunicava com a coronária nativa.

Conclusão: O implante de artéria torácica interna em miocárdio viável pode ter lugar no arsenal terapêutico da revascularização miocárdica, em casos especiais. Agora, baseados nos erros do passado, podemos selecionar os pacientes e obter melhores resultados. É uma técnica pouco estudada, que deveria ser mais usada, assim possibilitando uma avaliação mais precisa de sua eficácia.

DESCRITORES: Artérias torácicas, implante, métodos. Revascularização, miocárdico, métodos. Procedimentos cirúrgicos cardíacos, métodos. Vasos coronários, cirurgia. Artérias torácicas, cirurgia.

\section{INTRODUÇÃO}

O tratamento da doença coronária já há muito vem sendo tentado por diversos autores.

Em 1933, WEARNS et al. (1) descreveram a característica única da microanatomia do miocárdio, a presença de sinusóides que formam uma vasta rede vascular entre o sistema venoso e o arterial desse músculo, sendo uma importante fonte de suporte sangüíneo para as células miocárdicas.

Logo em seguida, baseados neste estudo, apareceram, então, várias tentativas de restaurar indiretamente o fluxo sangüíneo para o miocárdio isquêmico. Técnicas, como fixação do pedículo do

Trabalho realizado no Serviço de Cardiologia do Hospital São José do Avaí. Itaperuna, RJ, Brasil. Apresentado ao 26- Congresso Nacional de Cirurgia Cardíaca. Fortaleza, CE, 8 a 10 de abril de 1999.

*Do Serviço de Cardiologia do Hospital São José do Avaí.

Endereço para correspondência: Gladyston Luiz Lima Souto. Rua Lenira Tinoco Calheiros, 242. Centro. Itaperuna, RJ, Brasil. CEP 28.300-000. Tel: (24) 824-4191.e-mail:gladyston@tic.psi.br 
Souto G L L, Caetano C S, Souza J B, Paula A G, Souto H B, Coelho S B P, Reis E S V, Teixeira M A, Carvalho M R M, Botelho A C - Implante intra-miocárdico de artéria torácica interna: cirurgia de Vineberg. Rev Bras Cir Cardiovasc 2000; 15(4): $285-92$.

músculo peitoral no coração, talcagem no pericárdio $(2,3)$, fixação do omento ${ }^{(4)}$, ligadura do seio coronário ou das grandes veias cardíacas $(5,6)$ e acupuntura miocárdica (7), tiveram sucesso limitado.

Vineberg(8), em 1946, tentou levar o fluxo sangüíneo para essa rede vascular miocárdica pelo implante da artéria torácica interna esquerda sangrando dentro do miocárdio. Embora a cirurgia de Vineberg tenha se tornado conhecida, não era vista com simpatia pela comunidade de cardiologistas e cirurgiões. Seus benefícios fisiológicos foram inconsistentes e por anos foi documentada em relatos esporádicos. Porém, com o advento da angiografia desenvolvida por SONES ${ }^{(9)}$, tornou-se possível demonstrar que esse procedimento de fato aumentava a perfusão sangüínea ao músculo cardíaco. Esse reconhecimento chegou tarde, pois logo apareceram os métodos de revascularização direta do miocárdio, com resultados muito mais efetivos e rápidos.

Hoje, o tratamento da insuficiência coronária tem sido realizado com indicações e resultados já estabelecidos tanto através da operação com ponte de safena ou enxertos arteriais, utilizando as artérias torácica interna, radial, epigástrica inferior, gastroepiplóicas $(10,11)$, ou pelos vários procedimentos hemodinâmicos, como angioplastia transluminal coronária, stent, rotablator e laser (12-14).

Porém, existem casos em que se torna impossível o tratamento tradicional, devido ao grande acometimento aterosclerótico das coronárias, fazendo-se necessário uso de procedimentos, como a endarterectomia coronária, cujos resultados são discutíveis $(15,16)$. Ainda há casos que até a endarterectomia é impossível de se realizar, embora ainda haja nessas áreas miocárdio viável.

Recentes publicações vêm tornando cada vez mais claros os benefícios do implante da artéria torácica interna (ATI) no miocárdio em pacientes isquêmicos ${ }^{(17-23)}$, reforçando a crença de Vineberg. Isso poderia dar mais crédito ao seu trabalho e não o deixar relegado ao "status" de curiosidade histórica (20).

Esses fatos nos levaram a reviver a cirurgia de Vineberg em casos especiais e os nossos resultados são apresentados neste trabalho.

\section{CASUÍSTICA E MÉTODO}

Nos últimos 5 anos, foram operados 10 pacientes, nos quais foi implantada artéria torácica interna no miocárdio. As idades variaram entre 42 e 73 anos, sendo 6 pacientes do sexo masculino e 4 do feminino.

Todos os pacientes tiveram indicação para revascularização direta do miocárdio. As operações foram realizadas através de esternotomia mediana com auxílio de circulação extracorpórea (CEC) e uso de cardioplegia anterógrada sangüínea normotérmica intermitente de baixo volume.

Após a verificação da inviabilidade de revascularização direta na área das artérias coronárias interventricular anterior (IVA) e diagonais na parede ântero-lateral do ventrículo esquerdo (VE), da dificuldade da endarterectomia, constatada a viabilidade miocárdica da área e feitos os enxertos nas outras artérias que apresentavam lesões, foi feita uma pequena incisão com bisturi lâmina 11 próxima à ponta do coração lateralmente à IVA. Através desta incisão introduz-se uma pinça no sentido da ponta para a base do coração, na região ântero-lateral do VE. Essa pinça (usamos um clamp infantil vascular longo) é introduzida delicadamente no miocárdio, com cuidado para não atingir a luz do VE, e aflora no epicárdio após um trajeto de aproximadamente $8 \mathrm{~cm}$ dentro do músculo. A ATI, por sua vez, previamente dissecada e embebida em solução com papaverina, é esqueletizada distalmente num comprimento aproximado ao tamanho do túnel construído no miocárdio. Após isso, ela tem seus ramos cortados rentes às suas origens e sua extremidade distal ligada. A ATI é, então, puxada para dentro do túnel pelo clamp, de forma que o segmento esqueletizado com os ramos sangrando fique totalmente dentro do túnel miocárdico (Figura 1). A incisão da ponta do miocárdio é, então, fechada, fixando-se a parte distal da ATI neste local. A aorta é despinçada e, após iniciada a atividade contrátril do coração, retira-se o clamp da ATI e se observa se há sangramento no orifício de entrada da ATI no miocárdio. Em caso afirmativo, uma sutura em bolsa pode ser aplicada de maneira extremamente cuidadosa, evitando-se angustiar a ATI. É feito, então, a retirada da CEC de maneira habitual e, após cuidadosa revisão da hemostasia, o paciente é fechado de maneira rotineira.

\section{RESULTADOS}

Os pacientes submetidos ao implante de ATI tiveram boa evolução no pós-operatório, sem qualquer repercussão clínica ligada ao método. Tiveram alta hospitalar entre o 6- e 10- dia de pós-operatório.

Os 10 pacientes permaneceram assintomáticos a médio prazo e 9 deles foram reestudados entre 


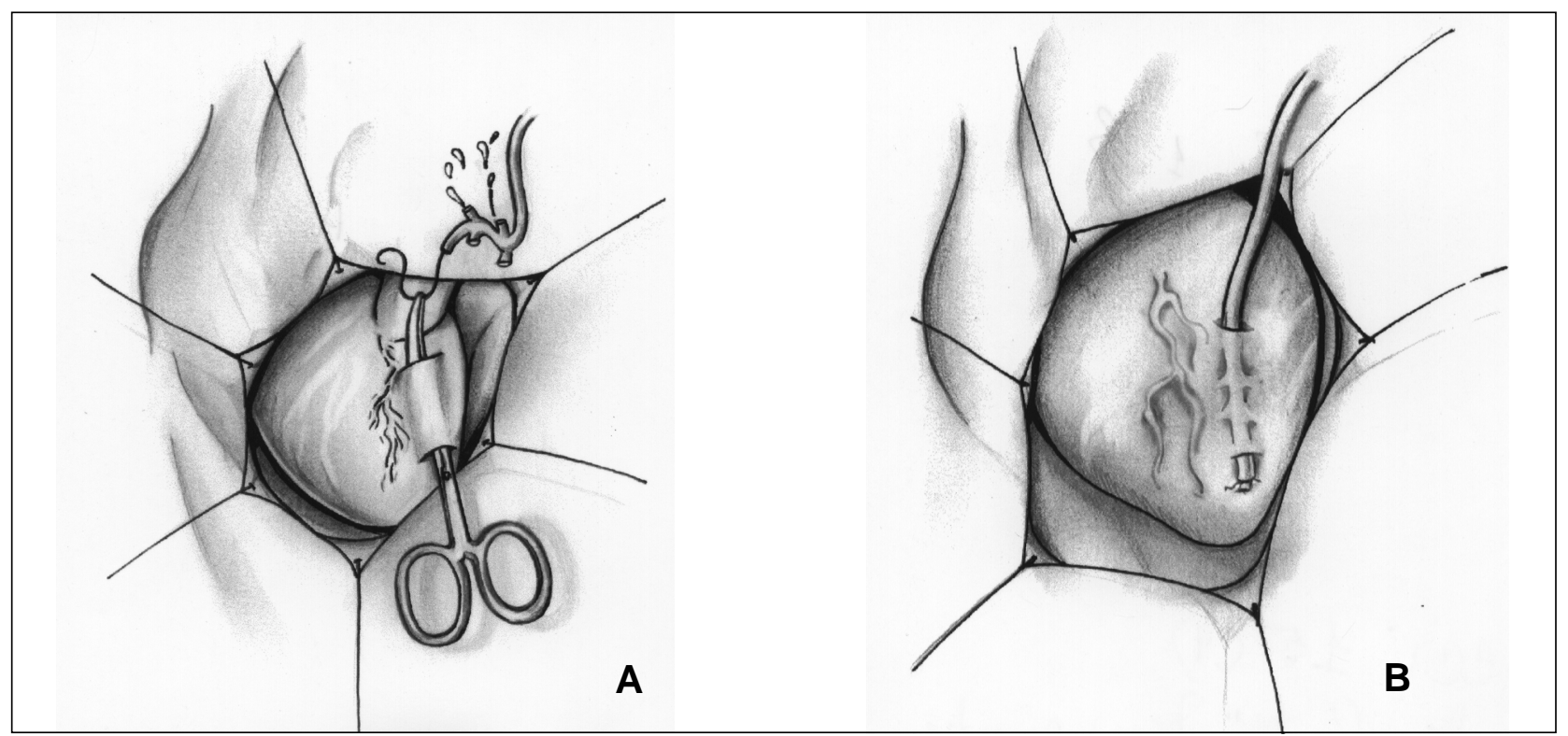

Fig. 1-Representação esquemática do implante de artéria torácica interna. A: Feitura do túnel no miocárdio. B: artéria torácica interna já implantada.

QUADRO 1

\begin{tabular}{rccccc}
\hline PACIENTE & IDADE & SEXO & $\begin{array}{c}\text { DATA } \\
\text { OPERAÇÃO }\end{array}$ & REESTUDO & $\begin{array}{c}\text { TEMPO DE } \\
\text { IMPLANTAÇÃO }\end{array}$ \\
\hline 1 & 71 & $\mathrm{~F}$ & $03 / 08 / 94$ & $07 / 04 / 95$ & $08 \mathrm{meses}$ \\
2 & 58 & $\mathrm{M}$ & $08 / 02 / 96$ & $06 / 11 / 96$ & $09 \mathrm{meses}$ \\
3 & 42 & $\mathrm{~F}$ & $05 / 01 / 95$ & $06 / 12 / 96$ & $23 \mathrm{meses}$ \\
4 & 46 & $\mathrm{M}$ & $14 / 10 / 96$ & $06 / 12 / 96$ & $45 \mathrm{dias}$ \\
5 & 66 & $\mathrm{M}$ & $07 / 02 / 95$ & $07 / 12 / 96$ & $22 \mathrm{meses}$ \\
6 & 65 & $\mathrm{~F}$ & $09 / 06 / 96$ & $09 / 09 / 97$ & $15 \mathrm{meses}$ \\
7 & 44 & $\mathrm{M}$ & $07 / 03 / 96$ & $12 / 09 / 97$ & $18 \mathrm{meses}$ \\
8 & 69 & $\mathrm{M}$ & $08 / 05 / 96$ & $10 / 10 / 97$ & $17 \mathrm{meses}$ \\
\hline 9 & 69 & $\mathrm{~F}$ & $15 / 03 / 97$ & $06 / 05 / 98$ & $14 \mathrm{meses}$ \\
\hline
\end{tabular}

45 dias e 23 meses após a operação (Quadro 1). O estudo angiográfico da ATI evidenciou patência desses vasos em todos os casos. Conforme o intervalo de tempo entre o implante e o estudo angiográfico, observamos extensa rede vascular entre elas e o miocárdio adjacente (Figura 2). A ventriculografia mostrou melhora da função ventricular em todos os pacientes (Figura 3).

\section{COMENTÁRIOS}

Muitos fatores são responsáveis pelo sucesso da cirurgia de Vineberg em termos de patência da ATI implantada ou desenvolvimento de circulação colateral. SEWELL \& DAVALOS ${ }^{(24)}$ afirmaram que a chave para a formação da circulação colateral é a diferença de pressão entre a rede vascular miocárdica e a ATI implantada.

CASE et al. ${ }^{(25)}$ concordaram com essa afirmativa e acrescentaram que há necessidade de obstrução de $\pm 90 \%$ da artéria coronária nativa e que já existam colaterais na área. Seus implantes estavam patentes em $95 \%$ dos pacientes, quando as coronárias da região estavam com mais de $75 \%$ de obstrução. Em comparação, encontraram somente $25 \%$ de patência quando o grau de obstrução era menos de $75 \%$ da luz da coronária. LANGSTON et al. (26) postularam que, além do gradiente entre os dois sistemas, é de igual importância a capacidade 


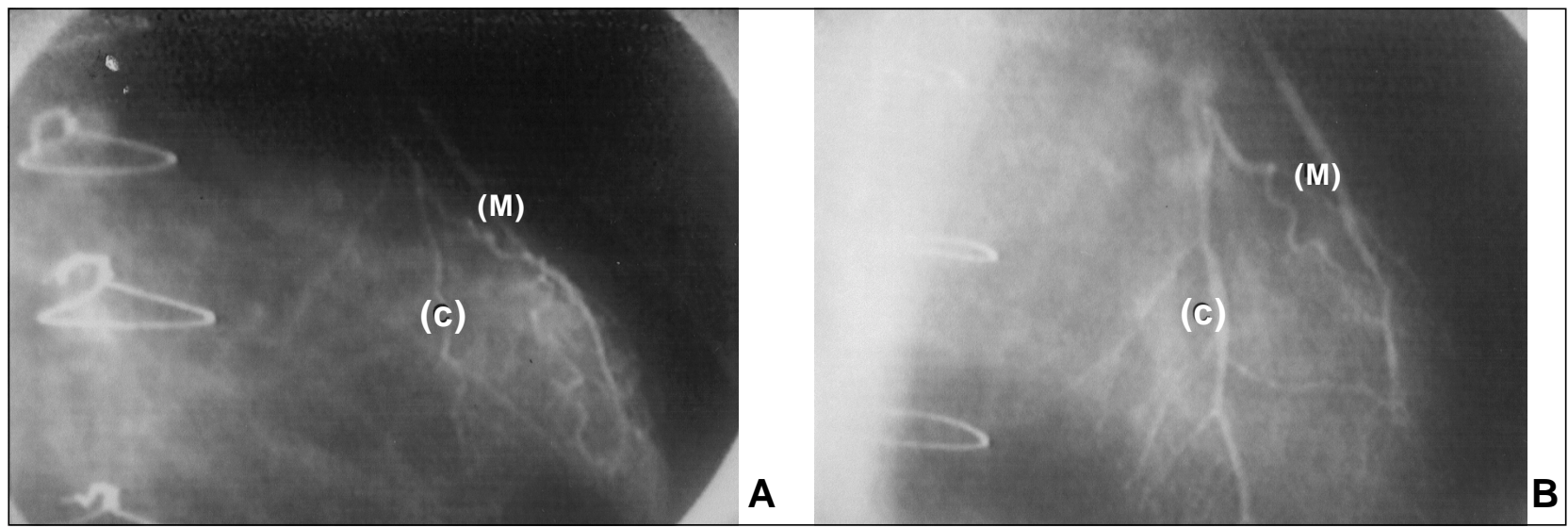

Fig. 2 - Reestudos angiográficos de pacientes com artéria torácica interna implantada (M). Vasta comunicação entre as artérias torácicas internas e artérias coronárias nativas (C). A: paciente com 15 meses. B: paciente com 18 meses.

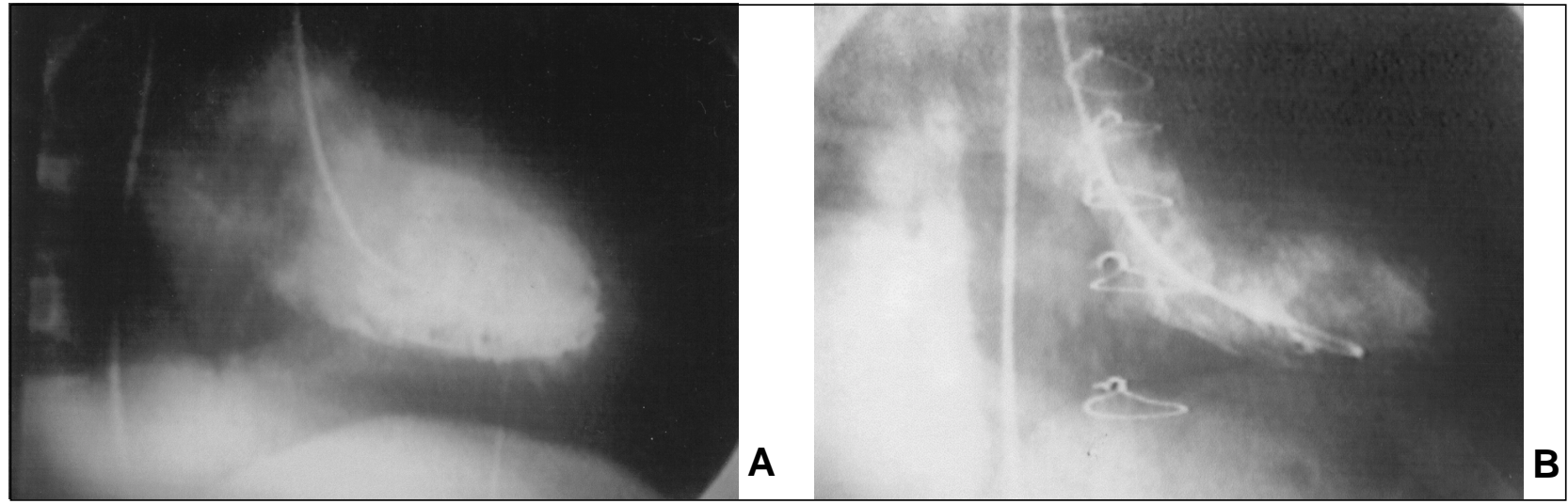

Fig. 3 - A: Ventriculografia no pré-operatório (sístole) de implante de artéria torácica interna implantada. B: Ventriculografia 14 meses após a operação.

de desenvolvimento da circulação colateral que varia de um paciente para o outro. FAVALORO et al. (27) afirmaram que a preexistência de circulação colateral é um fator importante (28).

Em relação ao alívio dos sintomas, alguns autores $(29,30)$ foram incapazes de correlacionar sintomas com a patência do implante. OCHSNER et al. (31) acharam que o desaparecimento dos sintomas só é indicador de patência do implante em pacientes trivasculares graves, quando esses têm evidente melhora clínica.

A expectativa de vida após o implante é outra questão: DIETHRICH et al. (32) compararam um grande número de pacientes que receberam implante de ATI com pacientes que não receberam, mas com doença coronária similar. A sobrevida foi de $70 \%$ nos pacientes operados (contando com as mortes na operação) e de $58 \%$ nos não operados. GORLIN \& TAYLOR (33) verificaram a redução de
$50 \%$ de infarto do miocárdio em pacientes operados, quando comparados ao grupo não operados que tinham doença coronária similar. KASSEBAUM et al. (34) evidenciaram melhora evidente à eletrocardiografia nos pacientes que receberam implantes de ATI.

Talvez a dificuldade de relacionar a clínica com a patência do implante seja porque esses doentes estudados só receberam implantes de ATI em uma região do miocárdio, ficando as outras áreas desprotegidas e nas quais a doença evoluiu. Não havia, na época, uma divulgação de revascularização direta do miocárdio e nem a sua avaliação pela cinecoronariografia, embora já fossem usadas.

DOBELL (18) afirmou que a patência do implante demonstrado angiograficamente não evidencia um bom resultado da operação, pois há casos em que o implante está patente e não é visto um bom fluxo sangüíneo para o miocárdio, ou mesmo não apre- 
Souto G L L, Caetano C S, Souza J B, Paula A G, Souto H B, Coelho S B P, Reis E S V, Teixeira M A, Carvalho M R M, Botelho A C - Implante intra-miocárdico de artéria torácica interna: cirurgia de Vineberg. Rev Bras Cir Cardiovasc 2000; 15(4): $285-92$.

senta nenhum "blush" de contraste dentro do miocárdio e, em outros, se visualiza uma intensa circulação colateral.

Isso levantou controvérsias sobre a efetividade do método, porém, em quase todos esses estudos na época em que esses pacientes foram operados, não houve uma avaliação perfeita na seleção para o implante, como a exclusão de zonas não isquêmicas ou com fibrose.

DOBELL (18) também cita que uma boa prova da efetividade do implante é o comentário de vários cirurgiões que, quando foram reoperar um paciente para revascularização direta, cuja primeira operação tenha sido o implante de ATI, após o pinçamento da aorta, o coração continuava a bater somente parando quando a ATI implantada fora pinçada.

Atualmente, tem-se estudado bastante uma excelente idéia para atingir a rede vascular do miocárdio, que é o Laser Transmiocárdico (35-39). Esse tem a finalidade de criar canais sem fibrose a partir do ventrículo esquerdo, a fim de aumentar a revascularização do miocárdio. Talvez seja esse um dos caminhos para a revascularização perfeita do miocárdio, porém essa rede já foi atingida por outro caminho que é o implante da ATI, sem uso de máquinas sofisticadas, com benefícios já comprovados (40-44). Por que não estudá-los mais, para consagrar essas afirmativas ou não ?

Recentemente, começaram a aparecer estudos da longa sobrevida de pacientes com implante de ATI. HAYWARD RH et al. (17), em 1991, concluíram que o implante de ATI tem um lugar no armamento cirúrgico da revascularização do miocárdio e que a colateralização desenvolvida pelo implante tem a mesma durabilidade de quando se faz a anastomose direta da ATI nas artérias coronárias, sendo seus efeitos a longo prazo similares, diferentemente dos enxertos venosos, que são bons imediatamente, mas com limitada durabilidade.

Em 1992, TOPAZ et al. (19) confirmaram também esses benefícios da ATI implantada.

NASU et al. (22), em 1996, avaliaram o volume e a reserva do fluxo da ATI implantada pela ecodopplercardiografia em pacientes após 23 anos de operação, chegando à conclusão de que o modelo de fluxo é bifásico, como o fluxo coronário normal, e que a reserva de fluxo desses implantes foi apenas $30 \%$ menos do que a reserva de fluxo de uma ATI anastomosada na coronária que apresentava $90 \%$ de obstrução.

Em 1999, THOMAS (23) afirmou que as idéias de Vineberg em relação ao implante de ATI podem formar a base do futuro tratamento da doença coronária.

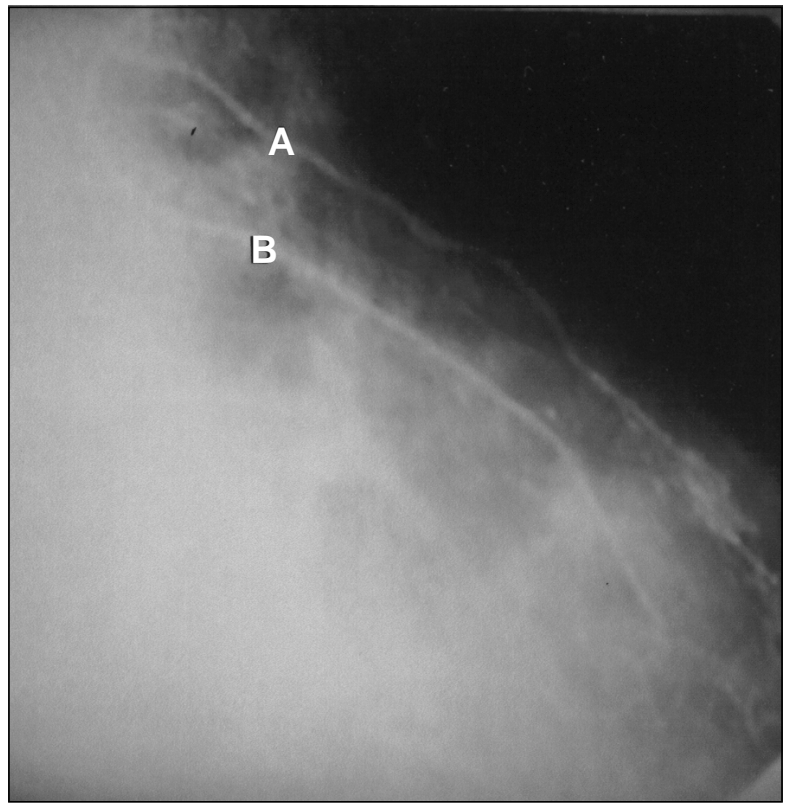

Fig. 4 - Reestudo angiográfico do paciente com 9 meses de artéria torácica interna implantada implantada. A: ATI implantada. B: Grande desenvolvimento do ramo da artéria torácica interna implantada (pericárdio frênico).

Todos os nossos pacientes foram para operação com a finalidade de se fazer enxerto para as coronárias, embora soubéssemos do comprometimento arterosclerótico importante das artérias interventricular anterior e diagonais. Dez deles apresentavam artérias mais comprometidas. Segundo a angiografia, todos eles já apresentavam uma rede de circulação colateral. Optamos pela realização do implante por acharmos que a endarterectomia seria difícil e de resultados incertos.

Todos os implantes foram feitos no território da artéria interventricular anterior e diagonais, pela técnica proposta por Vineberg. Não havia qualquer sinal de fibrose da região e as outras artérias doentes foram tratadas com ponte de safena.

Em um dos nossos pacientes a dissecção da ATI com ligadura dos ramos não foi completa. Foi deixado um dos seus ramos pericárdico frênico que, no pós-operatório, mostrou-se aumentado devido à elevação da resistência temporária da ATI dentro do miocárdio. Embora, o resultado tenha sido bom, poderia ser melhor. Portanto, a dissecção perfeita da ATI com ligadura de todos os seus ramos é essencial para evitar esses desvios de fluxo (Figura 4).

Embora sabendo que os resultados mais efetivos do implante de ATI começam a aparecer depois de aproximadamente 3 meses, quando a circulação colateral já se formou (45), em um de nossos casos estudados com 45 dias após o implante, já observamos um "blush" na região (Figura 5). Em alguns 


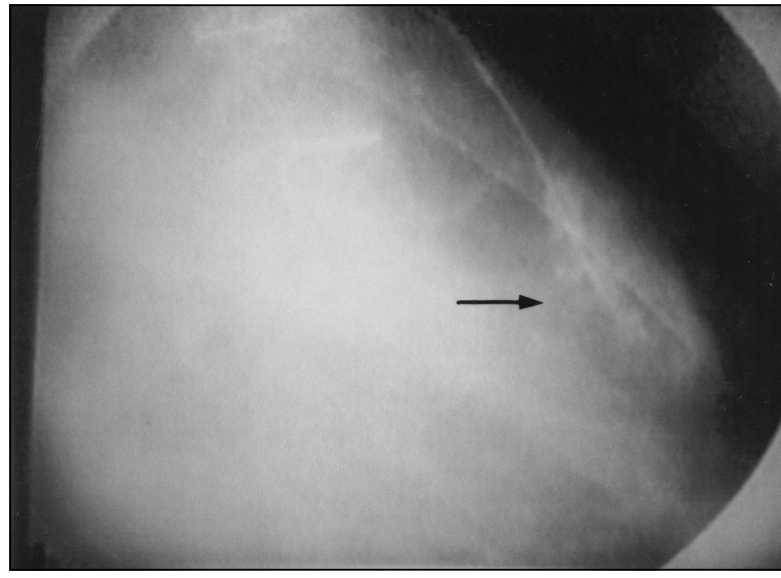

Fig. 5 - Reestudo angiográfico do paciente com 45 dias da artéria torácica interna implantada implantada, observa-se um "blush" dentro do miocárdio (seta)

de nossos pacientes houve uma ligeira alteração visível à eletrocardiografia (supra de ST) correspondendo à área implantada, porém desapareceu logo.

Podemos dizer que o implante da artéria ATI no miocárdio deve seguir os seguintes pontos básicos: 1) implantar em região que exista obstrução da coronária superior a $90 \%$; 2) coronária cuja coloca- ção de enxerto é inviável pelo grande acometimento aterosclerótico; 3) quando o resultado da endarterectomia é duvidoso; 4) existência de uma rede de circulação colateral; 5) inexistência de fibrose na região e presença de miocárdio viável.

Pelos nossos resultados, apesar do pequeno número de casos, fomos estimulados a pensar e a crer mais no implante de ATI e realizá-lo, se possível, com mais freqüência. Se essa crença se difundisse, teríamos mais pacientes para podermos utilizar os métodos modernos de avaliação da sua efetividade e, talvez fosse o caminho para ampliarmos a indicação dessa técnica no futuro.

\section{CONCLUSÃO}

O implante da ATI em miocárdio viável, pode ter lugar no arsenal terapêtico da revascularização do miocárdio, em casos especiais.

Agora, baseados nos erros do passado, podemos selecionar melhor os pacientes e obter melhores resultados. É uma técnica pouco estudada, que deveria ser mais usada, possibilitando, assim, uma avaliação mais precisa de sua eficácia.

RBCCV 44205-514

Souto G L L, Caetano C S, Souza J B, Paula A G, Souto H B, Coelho S B P, Reis E S V, Teixeira M A, Carvalho M R M, Botelho A C - Internal mammary artery myocardial implantation: Vineberg's procedure. Rev Bras Cir Cardiovasc 2000; 15(4): 285-92.

ABSTRACT: Objetive: To demonstrate our experience with the myocardial revascularization using the implantation of left internal mammary artery. This procedure was proposed by Vineberg, in 1946.

Material and Methods: From August 1994 to September 1998, 10 patients were operated on, all of them had the left internal mammary artery implanted in the anterolateral part of the wall of the left ventricle and all of them had a saphenous vein bypass graft. The age of the patients ranged from 42 years to 73 years with the prevalence of males. All patients had a diffuse and severe coronary artery disease mainly of the interventricular (descending) anterior artery, but with myocardial viability. The indication for internal mammary implantation was made during the procedure, because the techniques as saphenous vein bypass or endarterectomy were not considered applicable to these cases.

Results: All the patients were very well in the post operative period; from 45 days to 23 months, coronariography study were performed on nine patients, showing the implanted internal mammary artery arborizing and communicating with the native coronary arteries.

Conclusion: The internal mammary artery implanted in a viable myocardium should have its place on myocardial revascularization treatment, in selected cases. Nowadays, observing the misinterpretation on the past papers, we can select the patients with appropriate indication for this technique, obtained good results. The Vineberg technique needs more experience to define the evaluation of its real effectiveness.

DESCRIPTORS: Thoracic artery implantation, methods. Myocardial revascularization, methods. Cardiovascular surgical procedure, methods. Cardiac surgical procedures, methods. Coronary vessels, surgery. Thoracic arteries, surgery. 
Souto G L L, Caetano C S, Souza J B, Paula A G, Souto H B, Coelho S B P, Reis E S V, Teixeira M A, Carvalho M R M, Botelho A C - Implante intra-miocárdico de artéria torácica interna: cirurgia de Vineberg. Rev Bras Cir Cardiovasc 2000; 15(4): $285-92$.

\section{REFERÊNCIAS BIBLIOGRÁFICAS}

1 Wearn J T, Mettier S R, Klumpp T G, Zschiesche L J - The nature of the vascular communications between the coronary arteries and the chambers of the heart. Am Heart J 1933; 9: 143-64.

2 Beck C - The development of a new blood supply to the heart by operation. Ann Surg 1935; 2: 102: 801.

3 Beck C - Further date on the establishment of a new blood supply to the heart by operation. $J$ Thorac Surg 1936; 5: 604.

4 O'Shaughnessy L - An experimental method of providing a collateral circulation to the heart. Br J Surg 1935: 23: 665 .

5 Gross L, Blum L, Silverman G - Experimental attempts to increase the blood supply to the dog's heart means of coronary sinus occlusion. J Exp Med 1937; 65: 91.

6 Beck C - The coronary operation. Am Heart J 1941; 22: 531 .

7 Sen P K, Udwadia T E, Kinare S G, Parukar G B Transmyocardial acupuncture: a new approach to myocardial revascularization. J Thorac Cardiovasc Surg 1965; 50: 181-9.

8 Vineberg A M - Development of anastomosis between coronary vessels and transplanted internal mammary artery. Can Med Assoc J 1946; 55: 17-9.

9 Sones F M Jr - Acquired heart disease: symposium on present and future of cineangiocardiography. $A m \mathrm{~J}$ Cardiol 1959; 3: 710.

10 Acar C, Jebara V A, Portoghese M et al. - Revival of the radial artery for coronary artery bypass grafting. Ann Thorac Surg 1992; 54: 652-60.

11 Wilson $\mathrm{J}$ M \& Ferguson $\mathrm{J} \mathrm{J}$ - Revascularization therapy for coronary artery disease: coronary artery bypass grafting versus percutaneous transluminal coronary angioplasty. Tex Heart Inst J 1995; 22: 145-61.

12 Jones $\mathrm{R} \mathrm{H}$, Philips $\mathrm{H} \mathrm{R}$, Mark $\mathrm{D} B$ et al. - Long term survival benefit of $C A B$ and PTCA in patients with coronary artery disease. Annual Meeting of the American Association for Thoracic Surgery. 75 th 1995 (Abstracts).

13 Arie S, Garcia P, Brito F S, Caixeta A M, Soares P $R$, Horta P E - Angioplastia coronária com uso de Laser. Rev Soc Cardiol Estado de São Paulo 1995; 5: 552-9.

14 Souza A G M R, Pinto I, Tanajura L F et al. - O stent de Palmz Schatz para o tratamento da doença aterosclerótica obstrutiva coronária. Rev Soc Cardiol Estado de São Paulo 1995; 5: 560-5.

15 Christakis G T, Rao V, Fremes S E, Chen E, Naylor C D, Goldman B S - Does coronary endarterectomy adversely affect the results of bypass surgery? $J$ Card Surg 1993; 8: 72-8.

16 Minale C, Nikol S, Zander M, Uebis R, Effert S, Messmer BJ - Controversial aspects of coronary endarterectomy. Ann Thorac Surg 1989; 48: 235-41.

17 Hayward R H, Korompai F L, Knight W L - Long-term follow-up of the Vineberg internal mammary artery implant procedure. Ann Thorac Surg 1991; 51: 1002-3.

18 Dobell A R - Arthur Vineberg and the internal mammary artery implantation procedure. Ann Thorac Surg 1992; 53: 167-9.

19 Topaz O, Pavlos S, Mackall J A, Nair R, Hsu J - The Vineberg procedure revisited: angiographic evaluation and coronary artery bypass surgery in a patient 21 years following bilateral internal mammary artery implantation. Cathet Cardiovasc Diagn 1992; 25: 218-22.

20 Shrager J B - The Vineberg procedure: the immediate forerunner of coronary artery bypass grafting. Ann Thorac Surg 1994; 57: 1354-64.

21 Meester A, Leonard J, Chenu P, Marchandise B Myocardial revascularization using Vineberg's procedure: 23 years follow-up. Arch Mal Coeur Vaiss 1994; 87: 1247-8.

22 Nasu M, Akasaka T, Chikusa H, Shoumura T - Flow reserve capacity of left internal thoracic artery 23 years after Vineberg procedure. Ann Thorac Surg 1996; 61: 1242-4.

23 Thomas $\mathrm{J} \mathrm{L} \mathrm{-} \mathrm{The} \mathrm{Vineberg} \mathrm{legacy:} \mathrm{internal} \mathrm{mammary}$ artery implantation from inception to obsolescence. Tex Heart Inst J 1999; 26: 107-13.

24 Swell W H \& Davalos P - Factors influencing collateral arterial formation from pedicles on the surface of the hearts of dogs. Angiology 1962; 13: 231.

25 Case R B, Nasser M G, Crampton R S - Biochemical aspects of early myocardial ischemia. Am J Cardiol 1969; 24: 766-75.

26 Langston M F Jr., Kerth W J, Selzer A, Cohn K E Evaluation of internal mammary artery implantation. Am J Cardiol 1972; 29: 788-92.

27 Favaloro R G, Effer D B, Groves K L, Fergusson D J, Lozada J S - Double internal mammary arterymyocardial implantation: clinical evaluation of results in 150 patients. Circulation 1968; 37: 549-55.

28 Taylor W J \& Gorlin R - Objective criteria for internal mammary artery implantation. Ann Thorac Surg 1967; 4: $143-50$.

29 Aldridge H E, Mac Gregor D C, Lansdown E L et al. Internal mammary artery implantation for the relief of 
angina pectoris: a follow-up study of 77 patients for up to 13 years. Can Med Assoc J 1968; 98: 194.

30 Sethi G K, Scott S M, Takaro T - Myocardial revascularization by internal thoracic arterial implants: long term follow-up. Chest 1973: 64: 235-40.

31 Ochsner J L, Moseley P W, Mills N L, Bower P J - Longterm follow-up of internal mammary artery myocardial implantation. Ann Thorac Surg 1977; 23: 118-21.

32 Diethrich E B, Liddicoat J E, Kinard S A, De Bakey M $E$ - An analysis of operated and nonoperated patients with documented coronary arterial disease. $J$ Thorac Cardiovasc Surg 1969; 57: 115-25.

33 Gorlin R \& Taylor W J - Myocardial revascularization with internal mammary artery implantation: current status. JAMA 1969; 207: 907-13.

34 Kassebaum D G, Judkins M P, Griswold H E - Stress electrocardiography in the evaluation of surgical revascularization of the heart. Circulation 1969; 40 : 297-313.

35 Horvath K A, Smith W J, Laurence R G, Schoen F J, Appleyard R F, Cohn L H - Recovery and viability of an acute myocardial infarct after transmyocardial laser revascularization. J Am Coll Cardiol 1995; 25: 258-63.

36 Smith J A, Dunning J J, Parry A J, Large S R, Wallwork $J$ - Transmyocardial laser revascularization $J$ Card Surg 1995; 10: 569-72.

37 Frazier O H, Cooley D A, Kadipasaoglu K A et al. Myocardial revascularization with laser: preliminary findings. Circulation 1995; 92 (suppl): II 58-65.
38 Galantier M, Moreira G B, Bub R F, Galantier J, Buffolo E, Carvalho A C, Armaganijan D, Felter J Revascularização transmiocárdica a laser. Rev Bras Cir Cardiovasc 1996; 11: 67-74.

39 Mirhoseini M, Muckerheide M, Cayton M M Transventricular revascularization by laser. Lasers Surg Med 1982; 2: 187-98.

40 Vineberg A \& Jewett B L - Development of an anastomosis between the coronary vessels and transplanted internal mammary artery Can Med Assoc J 1947; 56: 609-14

41 Vineberg A M - Development of anastomosis between the coronary vessels and a transplanted internal mammary artery. J Thorac Surg 1949; 18: 839-50.

42 Vineberg A M \& Niloff $\mathrm{P} \mathrm{H}$ - The value of surgical treatment of coronary artery occlusion by implantation of the internal mammary artery into the ventricular myocardium: an experimental study. Surg Gynecol Obstet 1950; 91: 551-61.

43 Vineberg A, Afridi S, Sahi S - Direct revascularization of acute myocardial infarction by implantation of left internal mammary artery into infarcted left ventricular myocardium. Surg Gynecol Obstet 1975; 140: 44-52.

44 Vineberg A - Evidence that revascularization by ventricular internal mammary artery implants increases longevity: twenty four-year, nine month follow-up. J Thorac Cardiovasc Surg 1975; 70: 381-97.

45 Baird R J, Shah P A, Ameli F M - The initial flow throughan internal mammary artery implanted into the myocardium. J Thorac Cardiovasc Surg 1971; 61: 456-60. 